peroxide or formaldehyde, or with cyanide or azide (F. H. Sobels).

The question of the contributions of the direct and indirect effects to biological damage received some attention. The direct effect in polythene and dry bovine serum can be influenced by oxygen or temperature, but seems to be independent of small amounts of water ( $P$. Alexander). In contrast, it was claimed that the radiosensitivity of enzymes in wet yeast cells is $2-50$ times higher than in dry cells, suggesting a high contribution from the indirect effect (F. Hutchinson).

The respiratory system of mouse-liver slices is affected by doses higher than $10^{6} \mathrm{r}$. Sensitivity is determined by cytochrome $c$, which is destroyed by indirect action (B. Rajewsky, G. Gerber and fH. Pauly). Yoshida ascites cells $20 \mathrm{~min}$. after 20,000 r. show a drop in respiration and in aerobic and anaerobic glycolysis, and changing relative levels of adenosine di- and tri-phosphate. These differences become less pronounced at $40 \mathrm{~min}$. (G. Höhne, H. A. Künkel and $H$. Maass). Incorporation of $d l$-phenylalanine $-{ }^{14} \mathrm{C}$ into cell components of mouse liver is higher after 500 r. whole-body exposure (J. A. V. Butler, P. Cohn and A. R. Crathorn). The rate of incorporation of phosphorus-32 into rat thymus deoxyribonucleic acid is reduced to half within $3 \mathrm{~min}$. after $1,000 \mathrm{r}$. to the whole body (M. G. Ord and L. A. Stocken). Increased incorporation of tracers after irradiation may be due to reduced size of body pool ( $F$. Sherman and A. B. Almeida).

A single sub-sterilizing dose of $1,050 \mathrm{r}$. given to rabbit ovaries is less damaging to late primordial follicles than if given in fractions of $210 \mathrm{r}$. every $48 \mathrm{hr}$. (P. Desaive). The sterilizing dose for female mice, on the other hand, does not seem to be dependent on dose-rate, whether delivered at $2 \cdot 5 \mathrm{r}$. daily or in one exposure (H. and U. Langendorff).

In a session on genetic effects in Drosophila, H. $J$. Muller and I. I. Oster presented new evidence that true reversals of the original forked $\left(f^{\prime}\right)$ mutation can be induced by $\mathrm{X}$-rays. They believe that point mutations of whatever origin are indistinguishable in principle, and are due to qualitative chemical ehanges in the gene locus. Alkylating agents were reported to have produced a large number of point mutations in loci that have not been observed to mutate under $\mathrm{X}$-irradiation, and the distribution of chemically and X-ray induced mutations on the $X$-chromosome has been found to vary with the mutagen used $(O$. G. and M. J. Fahmy). Chromosomes of spermatids are at least twice as sensitive to the $\mathrm{X}$-ray induction of lethals and translocations as those of mature sperm in inseminated females (I. 1 . Oster). Crossing-over in spermatogonia can be induced by injection of formaldehyde (F. H. Sobels). Chromosome breaks, and possibly also intragenic changes, induced by $\mathrm{X}$-rays in mature sperm, are thought to undergo recovery if the sperm are retained for one day in the male (K. Nordback and C. Auerbach). At least five times as many small-effect detrimental mutations as recessive lethals are induced in male Drosophila by X-rays (K. G. Lüning and S. Johnsson). The reduced viability of flies receiving irradiated chromosomes is accompanied by a decreased rate of development, and it was suggested that growth-rate studies might be useful in assessing genetic damage due to radiation in mammals (G. Bonnier).

Two films were shown illustrating the effect on mitotic behaviour of irradiating parts of cells in tissue culture with $\alpha$-ray microbeams. Sensitivity increased by a factor of 10 from prophase to metaphase (M. I. Davis, I. Simon-Reuss and C. L. Smith), and irradiation of chromosomes was much more effective in producing anaphase and telophase abnormalities than irradiation of cytoplasm only (R. Munro). When roots of Vicia faba are irradiated with very high intensities of X-rays, in the range $600-1,200 \mathrm{r}$./min., a class of chromosome breaks becomes apparent which rejoin very rapidly and which may be due to breakage of ionic bonds. These breaks are to be distinguished from those others, regarded as due to the breakage of co-valent bonds, which remain open for a relatively long time (S. Wolff).

Under the appropriate chairmanship of Prof. $\AA$. Gustaffson, a special session met on the last day to discuss the application of the mutagenic effects of radiation to plant breeding. Facilities and techniques were described for the Brookhaven Laboratory (H. J. Curtis) and plans outlined for the improvement of the rice crop in India (A. R. Gopal-Ayengar). Of 4.27 radiation-induced mutations in soya beans, only 1 per cent seem to be of some economic value (M. Zacharias). In black currants, selection of mutated shoots from irradiated cuttings was reported to have given promising results (R. Bauer). A review was given of the work of the Swedish group in utilizing 'directed' mutations produced in barley by various physical and chemical agents (D. von Wettstein).

Michael EBERT.

Alima Howard

\section{POPULATION GENETICS}

A SYMPOSIUM on population genetics, with particular reference to stability of development, was held on the afternoon of Monday, September 3, at a joint session of Sections $D$ (Zoology) and $K$ (Botany) of the British Association in Sheffield. It was opened with a paper by Dr. J. M. Thoday entitled "Environmental Heterogeneity and the Genetic Architecture of Populations". Dr. Thoday began by pointing out that one of the most remarkable discoveries of recent biology was the great diversity of genotypes to be found in any population of out-breeding species. Comparative uniformity of individual morphology is mediated by a wide range of genotypes, each highly heterozygous and compounded of chromosomes many of which, though successful in heterozygous combinations, are deleterious, or even lethal, if made homozygous. This great genetic heterogeneity of populations is generally held to find its explanation as an adaptation permitting populations to survive change of their environment. Nevertheless, the heterozygosity on which this individuality depends involves a price, for it necessarily involves the production of some segregants poorly adapted to contemporary environmental conditions and, hence, involves relative infertility. The out-breeding systems by which heterozygosity is maintained are also less secure than self-fertilization. Some scientists therefore find it difficult to believe that the long-term selection for heterozygosity can be powerful enough to over-ride the short-term selection for fertility, and therefore doubt whether it is sufficient as an explanation of the ubiquity of the cross-breeding systems that maintain heterozygosity. Dr. Thoday suggested that if we consider the nature of environment heterogeneity, we find reason to believe that selection for 
heterozygosity on a relatively short-term basis may be strong. Environmental heterogeneity may be classified as random, directional or cyclic, the latter being the type to which he gave most attention. If the period of the cycle is longer than generation time, then the cycle will involve selection for new genotypes each generation and periodic reversal of the direction of selection. In these circumstances, a population may be expected to become heterozygous for genes appropriate to peak and trough conditions of the environmental oycle. Further, a certain degree of heterozygous advantage is to be expected as a system buffering the population against loss of peak genes in trough conditions, and vice versa. This system is precisely that known to occur in populations of D. pseudoobscura studied by Dobzhansky. Superimposed on the system, and hence in a heterozygous context, selection will proceed further to produce individuals capable of withstanding a longer and larger proportion of the range of environmental conditions imposed by the cycle. Only the success of this form of evolution can permit the evolution of longer,generation times, and the end will be heterozygous individuals that can withstand the whole cycle and hence perhaps live for several cycles.

Dr. Thoday suggested that our finding that the ability of individuals to control their development despite environmental variation very often depends on heterozygosity may well be explicable in terms of such a history of adaptation to cyclic conditions which he attempted to show are ubiquitous, especially since spatial differentiation of the environment must produce cyclic conditions in time, as dispersal of seed, etc., works in both directions.

The remaining papers were all concerned with this ability of individuals to maintain organized 'normal' development despite internally or externally caused disturbances. Mr. J. A. Beardmore reported on the effects of a diurnal temperature cycle on stability as measured by bilateral asymmetry of sternopleural chreta number in Drosophila melanogaster. Two long inbred lines the inbreeding history of which had occurred at constant $25^{\circ}$ give more stable $F_{1}$ hybrids at $25^{\circ}$, the $\mathrm{F}_{2}$ flies being, if anything, more stable than the $F_{x}$. In the fluctuating temperature conditions (mean $25^{\circ}$, midnight $20^{\circ}$, midday $30^{\circ}$ ), the reverse result is found, the $F_{1}$ being less stable than the inbreds and the $F_{2}$, if anything, less stable than $F_{1}$. He also reported evidence that though laboratory flies from constant $-25^{\circ}$ rooms are usually less stable in the fluctuating than in the constant-temperature incubator, newly captured wild flies give offspring superior in the fluctuating condition, indicating this to be the nearer to a wild environment. Six months in constant laboratory conditions may be sufficient for populations to lose their relative adaptation to the fluctuating condition. Experiments on natural populations involving comparisons in constant temperatures have therefore to be interpreted with caution.

Mr. J. Maynard Smith followed with a paper entitled "Developmental Flexibility in Drosophila". He began by stressing the point that the degree to which the development of organisms is under control or stabilized is not always to be measured by the degree of uniformity of the end product. Sometimes fitness may require different phenotypes in different environments. The superior capacity of outbreds, as compared with inbreds, is usually deemed demonstrated when it is shown that the outbreds are more uniform than corresponding inbreds; but when the situation is such that optimum phenotype varies with environment, then the best-regulated development should lead to variation of phenotype corresponding to variation of environment, and in these conditions, if outbred individuals are superior to inbreds, they should vary more than inbreds.

Mr. Maynard Smith described his attempt to test this hypothesis in Drosoplila subobscura. The character measured was the length of time for which adult flies could survive at $33.5^{\circ} \mathrm{C}$. in dry air. Three inbred lines and the three hybrid populations between them have been tested. In all three hybrid populations, individuals which were kept at $25^{\circ} \mathrm{C}$. as larvæ and pupæ, and then at $15^{\circ} \mathrm{C}$. as adults for a week before testing, lived approximately $2 \cdot 5$ times as long as did individuals of the same genotype kept at $15^{\circ} \mathrm{C}$. throughout. Thus outbred individuals are capable of long-lasting acclimatization to high temperatures if they are kept at $25^{\circ} \mathrm{C}$. during the preadult stages. Of the three inbred lines, one showed no capacity for acclimatization, and the other two acclimatized significantly less than did the hybrids. Thus the capacity to withstand exposure to high temperatures is a phenotypic character which can be modified adaptively in response to changed conditions during development, to a greater extent in outbred than in inbred individuals. In this particular case, therefore, the hypothesis outlined above has been confirmed.

The final paper was given by Dr. H. Rees on "The Stability of Meiosis in Inbred and Crossbred Rye". He said that when inbreeding is forced upon a species such as rye, which normally outbreeds, the progeny show inbreeding depression in relation to the chromosome mechanism much as they do for more familiar aspects of the phenotype. At meiosis one general consequence is a reduction in chiasma frequency. In some extreme cases when chromosomes are frequently unpaired and hence lost during cell division, this causes considerable pollen and egg infertility. Another result of inbreeding concerns the stability of plants in relation to chiasma frequencies. Thus between plants of identical genotype within inbred lines there is more variation in chiasma frequency than between plants of the same genotype within heterozygous families; under comparable experimental conditions the chiasma frequencies of homozygotes are less stable. Similarly, variation is greater between pollen mother cells within anthers of homozygotes than it is within anthers of heterozygotes. It has been found, however, that although homozygotes are generally less stable than heterozygotes, the instability cannot be explained in terms of the degree of homozygosis alone. Inbred lines equally homozygous may show different degrees of instability. Stability evidently must be related to the particular genotype.

Chiasma frequency, of course, to a large extent determines the degree of gene recombination and hence the variability in outbreeding populations. The average chiasma frequency is known to be genotypically controlled. The above results make it clear also that the range of chiasma frequencies within an anther (that is, the degree of 'instability') is also subject to the control of the genotype. From the population point of view this means, therefore, that the average amount of recombination through chiasma formation per plant may be adjusted by natural selection, and that the variety of the gametes, in respect of recombination, can also be adjusted adaptively. 